\title{
A retrospective review of 11 cases of villoglandular papillary adenocarcinoma of the uterine cervix and a review of the literature
}

\author{
LIPING ZHAO*, TIANMIN XU*, MANHUA CUI and ZEMING FU \\ Department of Gynecology and Obstetrics, The Second Hospital of Jilin University, Changchun, Jilin 130041, P.R. China
}

Received January 25, 2015; Accepted January 13, 2016

DOI: $10.3892 / 01.2016 .4172$

\begin{abstract}
Villoglandular papillary adenocarcinoma(VGPA) of the uterine cervix is a rare subtype of cervical adenocarcinoma (AC) that accounts for $3.7-4.8 \%$ of cervical AC. Compared with ordinary adenocarcinoma, VGPA is associated with a lower incidence, younger age and better prognosis. Treatment with fertility-preserving surgery is also possible. The present study analyzed cases treated in The Second Hospital of Jilin University (Changchun, Jilin, China) between January 2010 and December 2014. A total of 11 cases were selected; of these, 7 cases were pathologically confirmed. The remaining 4 cases were diagnosed as VGPA upon the pathological examination of the biopsy, but confirmed as another type of adenocarcinoma upon analysis of the surgical pathology. The median age of the patients whose pathology was confirmed post-operatively was 36 years, and all cases were positive for human papillomavirus (HPV). As all 7 patients had no requirement for further fertility, a radical hysterectomy was performed, with or without adjuvant therapy. The follow-up time ranged from 7-57 months, with a mean time of 29 months. There was no evidence of recurrence in any of the patients. All the patients remain alive to date. The results of the present study indicated that HPV infection is likely to be the cause of VGPA, and the prognosis of VGPA tends to be better compared with other types of adenocarcinoma. However, the number of studies that have reported cases of VGPA remains small, and all are retrospective analyses with short follow-up times. Therefore, physicians selecting treatment options for patients with VGPA should exercise caution.
\end{abstract}

Correspondence to: Professor Manhua Cui, Department of Gynecology and Obstetrics, The Second Hospital of Jilin University, 218 Ziqiang Street, Changchun, Jilin 130041, P.R. China

E-mail: cuimanhua55@126.com

*Contributed equally

Key words: uterine cervix, villoglandular, adenocarcinoma, diagnostic challenge, treatment

\section{Introduction}

Villoglandular papillary adenocarcinoma (VGPA) was first reported by Young and Scully in 1989 (1) and was classified as a histopathological entity of cervical cancer by the World Health Organization in 1994 (2). VGPA is a specific type of adenocarcinoma that is rarely identified by clinical diagnosis. To the best of our knowledge, $>150$ cases of VGPA of the cervix have been reported in the relevant literature. Human papillomavirus infection and oral contraceptive use are considered to be associated with VGPA $(3,4)$. The final diagnosis of VGPA depends on pathological confirmation. Histologically, VGPA is characterized by exophytic proliferation with long and slender papillary structures and a mild to moderate cellular atypia (5). There is no standard treatment for VGPA. It has been reported that fertility-preserving surgery may be considered if the tumor is at an early International Federation of Gynecology and Obstetrics (FIGO) stage, and if it not a mixed type adenocarcinoma of the uterine cervix that includes a villoglandular component $(6,7)$. Compared with ordinary adenocarcinoma, VGPA is associated with a lower incidence, younger age and better prognosis. These features have caused VGPA to gain more attention clinically. Treatment with fertility-preserving surgery is also now possible. In the present study, the clinical and pathological features of this neoplasm are retrospectively reviewed using cases from one institute, and the associated literature is also discussed.

\section{Patients and methods}

Clinical profiles of VGPA patients. All cases of VGPA identified by pre-operative biopsy or post-operative surgical pathology at The Second Hospital of Jilin University (Changchun, Jilin, China) between January 2010 and December 2014 were retrieved. A total of 11 cases were selected, however, patients whose post-operative diagnosis was of another type of adenocarcinoma or ordinary adenocarcinoma associated with VGPA were excluded. Therefore, 4 patients were excluded due to a pre-operative diagnosis of VGPA, but a final surgical pathological diagnosis of another type of adenocarcinoma or ordinary adenocarcinoma associated with VGPA. Only 7 cases were included in the present study. Among the 7 patients, $6(54.5 \%$; 6/11) were diagnosed with VGPA in the pre-operative and post-operative diagnoses. The remaining patient was pre-operatively diagnosed with another type of 
adenocarcinoma. The clinical profiles included data on patient age, presenting symptoms, history of oral contraceptive (OCP) use, human papillomavirus (HPV) infection, International Federation of Gynecology and Obstetrics (FIGO) staging, biopsy pathology, treatment, post-operative pathology, adjuvant therapy, lymph node (LN) metastasis, poor prognostic factors, follow-up and outcome.

\section{Results}

Clinical characteristics. The median age of the patients whose pathology was confirmed post-operatively was 36 years (range, 29-42 years). None of the patients had a history of smoking or OCP use. The most common symptom was contact bleeding (6 patients) and 1 patient exhibited abnormal vaginal bleeding. All patients underwent a pre-operative cervical biopsy. The predominant histological features of VGPA include a villoglandular architecture and mild to moderate cytological atypia and thus, VGPA was diagnosed in 6 patients and ordinary cervical adenocarcinoma in 1 patient. However, the post-operative diagnosis of all 7 patients was of VGPA, and all patients were positive for HPV.

In total, 6 patients $(85.7 \%)$ presented with FIGO stage IB1 disease and 1 with FIGO stage IIA1 disease (7). As all 7 patients were married with no desire for further fertility, a radical hysterectomy was performed, resulting in only 3 patients being left with one or both ovaries. Of the 7 patients, 2 possessed poor prognostic factors; 1 patient presented with invasive vascular infiltration, and the other with endometrial involvement and right ovarian borderline serous gonadal fibroma. Adjuvant therapy was being provided for 5 of the 7 patients. The follow-up time ranged from 7-57 months, with an average time of 29 months. There was no evidence of recurrence in any of the patients, and all patients remained alive at the time of writing this study. The details of the patients' characteristics, the pathology of the tumors and the management of the disease are shown in Table I.

The 4 women who were excluded from the present study were diagnosed with VGPA pre-operatively, but the final histological examination yielded a different diagnosis. These 4 patients (aged 44, 37, 48 and 47 years old) presented with abnormal vaginal bleeding or contact bleeding, and a punch biopsy was performed. The histological diagnosis was of VGPA (FIGO stages IB1, IB1, IB2 and IIA1). As the tumor was large and the women did not desire the preservation of fertility, all the patients underwent a radical hysterectomy with a double salpingo-oophorectomy and LN dissection. The final histology yielded a diagnosis of ordinary adenocarcinoma with negative nodes, not VGPA.

\section{Discussion}

VGPA of the uterine cervix is a rare subtype of cervical adenocarcinoma that accounts for $3.7-4.8 \%$ of all cervical AC $(1,8)$. Compared with other malignant cervical tumors, VGPA tends to occur in younger women, particularly those of reproductive age. Young and Scully (1) first reported 13 cases of VGPA with an average age of only 33 years old. The etiology of VGA remains unknown. Certain studies $(3,4)$ suggest a role for HPV infection in the pathogenesis of VGPA. Jones et al (3) reported

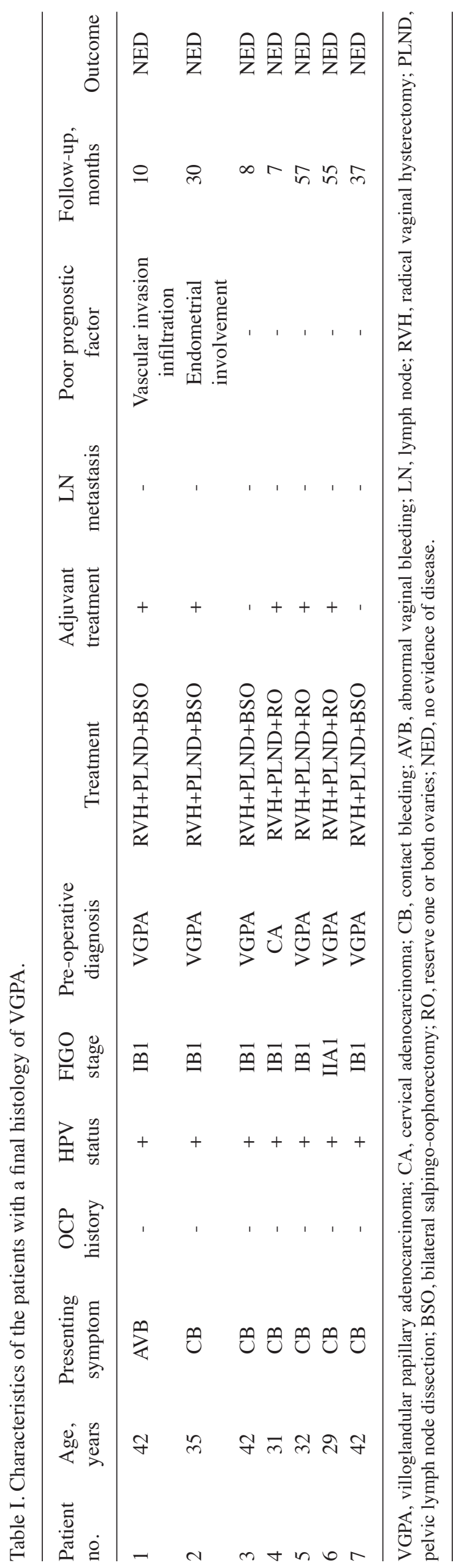


Table II. Profile of patients with villoglandular papillary adenocarcinoma associated with pregnancy.

\begin{tabular}{lccccr}
\hline First author, year & Age, years & FIGO stage & Follow-up & Outcome & Ref. \\
\hline Takai et $a l$, 2010 & 28 & IB1 & 44 months & NED & $(18)$ \\
Hurteau et $a l, 1995$ & 22 & IB & 14 months & NED & $(16)$ \\
Lavie et al, 2008 & 31 & IB1 & 18 months & NED & $(17)$ \\
Hoffman et al, 2001 & 28 & - & 24 months & NED & $(12)$ \\
Falcon et $a l, 2006$ & 34 & IB1 & 8 years & NED & $(14)$ \\
Korach et al, 2009 & 34 & IA1 & 10 years & NED & $(6)$ \\
\hline
\end{tabular}

-, data not available; NED, no evidence of disease; FIGO, International Federation of Gynecology and Obstetrics.

Table III. Incidence of lymph capillary space invasion and/or LN metastasis in the English literature.

\begin{tabular}{|c|c|c|c|c|c|c|c|}
\hline \multirow[b]{2}{*}{ First author, year } & \multirow{2}{*}{$\begin{array}{c}\text { Total } \\
\text { cases, } \mathrm{n}\end{array}$} & \multirow{2}{*}{$\begin{array}{l}\text { Cases of LN } \\
\text { metastasis, } n\end{array}$} & \multirow{2}{*}{$\begin{array}{l}\text { Cases of } \\
\text { recurrence, } n\end{array}$} & \multicolumn{3}{|c|}{ Cases of mortality } & \multirow[b]{2}{*}{ Ref. } \\
\hline & & & & Cases, $\mathrm{n}$ & Stage & Follow-up & \\
\hline Kaku et al 1997 & 7 & 2 & 1 & 1 & IIB & 46 months & (19) \\
\hline Jones et al 2000 & 12 & - & 2 & 1 & - & 79 months & (3) \\
\hline Korach et al 2009 & 9 & - & 1 & 1 & IB1 & 2 years & (6) \\
\hline Lataifeh et al 2013 & 28 & 2 & 5 & 5 & $\begin{array}{l}\text { IB2, IB, IIB, } \\
\text { IIIB, IIIB }\end{array}$ & 22.4 months $^{\mathrm{a}}$ & (20) \\
\hline Kim et al 2014 & 15 & 1 & 3 & 0 & - & - & (21) \\
\hline
\end{tabular}

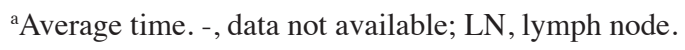
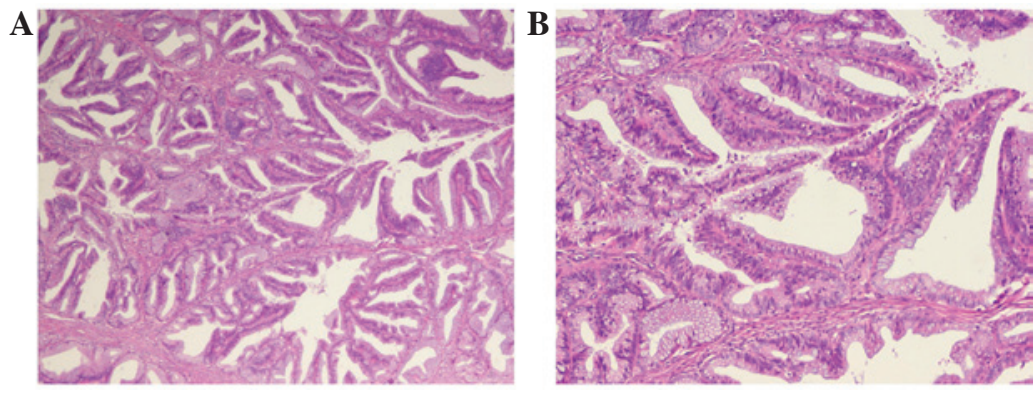

Figure 1. (A) Tumor displaying thin and tall, well-formed papillary structures (hematoxylin and eosin staining; original magnification, $\mathrm{x} 40$ ); (B) Large glandular and papillary structures with broad stroma (hematoxylin and eosin staining; original magnification, x100).

a study in which $15(62.5 \%)$ out of 24 VGPA patients were taking $\mathrm{OCP}$, with a duration of use (when known) ranging between 5 and 20 years; OCPs were considered to be associated with VGPA. In the present study, the median age of the 7 patients was 36.1 years, with a range of 29-42 years. None of the patients had a history of OCP use, but each case was positive for HPV infection. This test result indicates that HPV infection is more likely to be the cause of VGPA. The clinical manifestation of VGPA is similar to other types of cervical cancer with regard to abnormal vaginal bleeding, contact bleeding or abnormal vaginal secretion (1).

The final diagnosis of VGPA depends on pathological confirmation. As Young and Scully (1) reported that conservative treatment is feasible for VGPA, an accurate pre-operative diagnosis allows the opportunity for a number of VGPA-affected women to preserve their fertility. The main feature of VGPA is its villoglandular architecture. Each papilla in the villoglandular structures has a central pedicle that ranges in size and shape from short and thick to long and thin. The central pedicle contains a number of inflammatory cells (Fig. 1). The villoglandular structures are composed of stratified glandular cells, with mild to moderate nuclear atypia, and mitotic figures, with little or no budding from the villoglandular structures (9). A study by Macdonald et al (10) advised following the more recent changes in the definition of a villoglandular tumor rather than continuing to conform to a limited and conservative definition stating the presence of only villoglandular components, with no standard adenomatous or squamous features and no high-grade nuclear abnormalities. 
Among the 11 cases initially selected in the present study, only $54.5 \%(6 / 11)$ had the same pre-operative and post-operative diagnosis. Similarly, in a study by Korach et al (6), data were reviewed from the outpatient files of 12 patients who had been diagnosed with VGPA. The final pathology results confirmed a diagnosis of VGPA in only 9 of the patients. Of these 9 , only 2 had been correctly pre-operatively diagnosed, while in 3 , a benign or pre-malignant initial biopsy result had been determined, and in the remaining 4 patients, an invasive malignant tumor had been diagnosed, consequently necessitating a hysterectomy. The 3 non-VGPA patients were diagnosed with invasive cervical adenocarcinoma on the final histological report. Following integration of the data from the literature review and the results of the present study cases, it may be concluded that pre-treatment punch biopsy diagnosis has a low rate of diagnostic accuracy when compared with the final histological report. This may be attributed to two reasons: Firstly, forming an accurate pre-operative pathological diagnosis is difficult for VGPA. In $30 \%$ of cases, VGPA is associated with other types of invasive cancer $(1,11-14)$, which may have a significant impact on its prognosis. It is therefore important to remember that ordinary adenocarcinomas, including deeply invasive tumors, may have a papillary component and that VGPA should only be diagnosed in those lesions that are exclusively grade 1 and unassociated with an underlying conventional adenocarcinoma component. Secondly, the scope of a biopsy is limited, as it is unable to determine whether the entire tumor contains other invasive adenocarcinoma tissues or not. In addition, it is not easy to get to the basement membrane while taking a biopsy, so infiltration cannot be completely ruled out. Therefore, an excision or cone biopsy is occasionally required to establish a VGPA diagnosis when a punch biopsy is not sufficient.

Yamazawa et al (15) suggested that the recommendation of no further treatment may be acceptable for patients who require fertility preservation if the tumor is superficial with no involvement of the vascular space, or if the cone margins exhibit no residual disease. To date, only 6 cases of pregnancy associated with VGPA of the cervix have been reported (Table II). In 3 of these cases, successful conception was achieved following a conservative treatment for VGPA $(6,12,14)$. The 3 remaining cases were diagnosed during the pregnancy (16-18). Nonetheless, the management of cervical VGPA is considered to be a significant challenge. To date, $>150$ cases of VGA of the cervix have been reported in the English literature, with 12 cases of recurrence and 8 mortalities (Table III) $(3,6,19-21)$. In the clinical analysis of background data, the patients who experienced a poor outcome were closely associated with an advanced FIGO stage and LN metastasis. Of the mortalities, 5 patients had VGPA of stage IIB or greater. LN metastasis was reported in 5 patients, with 3 of these cases developing recurrence (2 patients succumbed to recurrence). As the current follow-up time of VGPA is short, a lack of data is available on the 5-year survival rate.

In the present study, the 7 patients with VGPA had no fertility requirements and exhibited a clinical stage of between IB1-IIA1; therefore, all the patients underwent a radical hysterectomy with or without adjuvant therapy. The follow-up time ranged from 7-57 months, with an average time of 28 months. All patients were alive with no evidence of recurrence at the time of writing this study.

In conclusion, VGPA usually occurs in females of reproductive age. Reproductive and endocrine treatment options are factors that must be considered in the treatment of affected patients, while the proposed conservative treatment for these women allows maintenance of fertility in patients who are young and wish to have children. However, as the number of studies reporting cases of VGPA remains small, and as all are retrospective analyses with a short follow-up time, more prospective randomized controlled trials are required to confirm the prognosis of the disease. Therefore, while choosing treatment options for patients with VGPA, data for disease stage, fertility requirements, follow-up conditions, and the presence of vascular and lymphatic metastasis should be integrated, which for a range of aspects, would aid in prudent decision-making.

\section{Acknowledgements}

The present study was supported by grants from the National Natural Science Foundation of China (nos. 81272875 and 81302242), the Outstanding PhD program of Norman Bethune College of medicine, Jilin University (no. 2013), the Jilin Science and Technology Fund (nos. 20110755, 20130102094JC and 20140204022YY) and the Basic Science Research Fund of Jilin University (no. 20142116).

\section{References}

1. Young RH and Scully RE: Villoglandular papillary adenocarcinoma of the uterine cervix. A clinicopathologic analysis of 13 cases. Cancer 63: 1773-1779, 1989.

2. Scully RE, Bonfiglio TA, Kurman RJ, Silverberg SG and Wilkinson EJ (eds). Uterine cervix. In: Histological Typing of Female Genital Tract Tumors. World Health Organization International Histological Classification of Tumors. 2nd edition. Springer-Verlag, New York, NY, 1994.

3. Jones MW, Kounelis S, Papadaki H, Bakker A, Swalsky PA, Woods J and Finkelstein SD: Well-differentiated villoglandular adenocarcinoma of the uterine cervix: Oncogene/tumor suppressor gene alterations and human papillomavirus genotyping. Int J Gynecol Pathol 19: 110-117, 2000.

4. An HJ, Kim KR, Kim IS, Kim DW, Park MH, Park IA, Suh KS, Seo EJ, Sung SH, Sohn JH, et al: Prevalence of human papillomavirus DNA in various histological subtypes of cervical adenocarcinoma: A population-based study. Mod Pathol 18: 528-534, 2005.

5. Khunamornpong S, Siriaunkgul S and Suprasert P:Well-differentiated villoglandular adenocarcinoma of the uterine cervix: Cytomorphologic observation of five cases. Diagn Cytopathol 26:10-14,2002.

6. Korach J, Machtinger R, Perri T, Vicus D, Segal J, Fridman E and Ben-Baruch G: Villoglandular papillary adenocarcinoma of the uterine cervix: A diagnostic challenge. Acta Obstet Gynecol Scand 88: 355-358, 2009.

7. Pecorelli S: Revised FIGO staging for carcinoma of the vulva, cervix, and endometrium. Int J Gynaecol Obstet 105: 103-104, 2009.

8. Heatley MK: Villoglandular adenocarcinoma of the uterine cervix-a systematic review of the literature. Histopathology 51: 268-269, 2007.

9. Nagai N, Hirata E, Kusuda T, Mukai K, Arihiro K and Ohama K: Villoglandular papillary adenocarcinoma of the uterine cervix responding to neoadjuvant chemotherapy with docetaxel and cisplatin: A case report. Int J Gynecol Cancer 15: 1187-1190, 2005.

10. Macdonald RD, Kirwan J, Hayat K, Herrington CS and Shawki H: Villoglandular adenocarcinoma of the cervix: Clarity is needed on the histological definition for this difficult diagnosis. Gynecol Oncol 100: 192-194, 2006. 
11. Jones MW, Silverberg SG and Kurman RJ: Well-differentiated villoglandular adenocarcinoma of the uterine cervix: A clinicopathological study of 24 cases. Int J Gynecol Pathol 12: 1-7, 1993.

12. Hoffman JS, Bazzurini L, Laird L, Murphy JC, Magriples U and Lewis J: Term delivery following conservative treatment for villoglandular papillary adenocarcinoma of the uterine cervix: Report of a case and analysis of the literature. Gynecol Oncol 81: 310-313, 2001

13. Dede M, Deveci G, Deveci MS, Yenen MC, Goktolga U, Dilek S and Gunhan O: Villoglandular papillary adenocarcinoma of the uterine cervix in a pregnant woman: A case report and review of literature. Tohoku J Exp Med 202: 305-310, 2004.

14. Falcon O, Garcia R, Lubrano A, Morín JC and Andujar M: Successful term delivery following conservative management for villoglandular papillary adenocarcinoma of the uterine cervix: A case report. Gynecol Oncol 101: 168-171, 2006.

15. Yamazawa K, Matsui H, Seki K, Mitsuhashi A, Kawamata Y, Shirasawa $\mathrm{H}$ and Sekiya S: Human papillomavirus-positive well-differentiated villoglandular adenocarcinoma of the uterine cervix: A case report and review of the literature. Gynecol Oncol 77: 473-477, 2000

16. Hurteau JA, Rodriguez GC, Kay HH, Bentley RC and Clarke-Pearson D: Villoglandular adenocarcinoma of the cervix: A case report. Obstet Gynecol 85: 906-908, 1995.
17. Lavie O, Segev Y,Peer G, Gutterman E, Sagie S and Auslnader R: Conservative management for villoglandular papillary adenocarcinoma of the cervix diagnosed during pregnancy followed by a successful term delivery: A case report and a review of the literature. Eur J Surg Oncol 34: 606-608, 2008.

18. Takai N, Hayashita C, Nakamura S, Narahara H and Matsumoto $\mathrm{H}$ : A case of villoglandular papillary adenocarcinoma of the uterine cervix diagnosed during early pregnancy followed by successful term delivery. Case Rep Med 2010 : $314547,2010$.

19. Kaku T, Kamura T, Shigematsu T, Sakai K, Nakanami N, Uehira K, Amada S, Kobayashi H, Saito T and Nakano H: Adenocarcinoma of the uterine cervix with predominantly villogladular papillary growth pattern. Gynecol Oncol 64: 147-152, 1997.

20. Lataifeh IM, Al-Hussaini M, Uzan C, Jaradat I, Duvillard P and Morice P: Villoglandular papillary adenocarcinoma of the cervix: A series of 28 cases including two with lymph node metastasis. Int J Gynecol Cancer 23: 900-905, 2013.

21. Kim HJ, Sung JH, Lee E, Ahn S, Song SY, Choi CH, Kim TJ, Kim BG, Bae DS and Lee JW: Prognostic factors influencing decisions about surgical treatment of villoglandular adenocarcinoma of the uterine cervix. Int J Gynecol Cancer 24: 1299-1305, 2014 